\title{
¿EXISTE UNA "MALLA DE SEGURIDAD” EN LA EUROPA DEL SUR? \\ La lucha contra la pobreza y la exclusión en España, Grecia, Italia y Portugal*
}

\author{
LUIS MORENO, MANOS MATSAGANIS, \\ MAURIZIO FERRERA y LUÍS CAPUCHA \\ Unidad de Políticas Comparadas (CSIC), Departamento de Económicas (University of Crete), \\ Università Bocconi (POLEIS), Departamento de Sociología (ISCTE)
}

PALABRAS ClAVE ADICIONALES

Asistencia Social, Europa del Sur, Exclusión

Social, Pobreza, Rentas Mínimas.

\section{ADDITIONAL KEYWORDS}

Social Assistance, Southern Europe, Social

Exclusion, Poverty, Minimum Incomes.

RESUMEN. El papel marginal de la asistencia social y la ausencia de programas de rentas mínimas han sido considerados como rasgos definitorios del modelo de bienestar de la Europa del Sur. Sin embargo, a lo largo de la década de los años 90 se han producido innovaciones significativas en este campo. El artículo lleva a cabo un análisis de los últimos desarrollos realizando un examen crítico de las experiencias de políticas contra la pobreza en España, Grecia, Italia y Portugal. Se arguye que el carácter fragmentario y "parcheado" de las mallas de seguridad (safety nets) en la Europa meridional se debe a un singular conjunto de circunstancias, las más relevantes de las cuales son el rol de las familias y una cierta morosidad de sus instituciones estatales. Un análisis de los perfiles nacionales revela que las nuevas políticas implementadas en los cuatro países persiguen solventar algunos de los desequilibrios históricos de este régimen de bienestar. En particular, los programas comprensivos de rentas mínimas están ahora vigentes en Portugal y en algunas Comunidades Autónomas

Este artículo se basa en los resultados del proyecto, Fighting poverty and social exclusion in southern Europe: dilemmas of organisation and implementation (FIPOSC), financiado por la Comisión Europea (HPSE-CT-2001-60020). Los autores agradecen a Ana Arriba, Francesca Bastagli, Teresa Bomba, Teresa Buil, Rita Fernandes, Giselas Matos y Stefano Sacchi por sus contribuciones y apoyo prestado, así como a todos los participantes en el seminario FIPOSC (Milán 23-24 Mayo, 2002). Luis Moreno agradece a la Secretaria de Estado de Educación y Universidades (PR2002-0200) su ayuda financiera durante la redacción de este artículo.

E-mail: Lmorfer@iesam.csic.es manos@econ.soc.uoc.gr maurizio.ferrera@uni-bocconi.it Luis.Capucha@iscte.pt

Revista Internacional de Sociología (RIS)

Tercera Época, $\mathrm{N}^{\circ} 36$, Septiembre-Diciembre, 2003, pp. 7-31. 
RIS

REVISTA INTERNACIONAL DE SOCIOLOCIA

№ 36 , Septiembre-Diciembre, 2003

LUIS MORENO, MANOS MATSAGANIS, MAURIZIO FERRERA Y LUIS CAPUCHA

españolas (aunque todas éstas disponen de algún tipo de actuación al respecto), mientras que una experimentación se ha llevado a efecto en Italia. A la luz de estas experiencias el artículo concluye que las mallas de seguridad y de protección social en la Europa del Sur existen, pero mantienen un débil diseño institucional asi como una limitada legitimidad y apoyo político.

\begin{abstract}
The marginal role of social assistance and the absence of minimum income programmes have long been thought to constitute defining characteristics of the southern European model of welfare. Nevertheless, over the 1990s significant innovations in this field have taken place. The article aims to contribute to the analysis of recent developments by critically examining the experience of anti-poverty policies in Greece, Italy, Portugal and Spain. It is argued that the 'patchiness' of safety nets in southern Europe is due to a unique set of constraints, the most relevant of which are the role of families and the 'softness' of state institutions. A review of national profiles reveals that new policies introduced in all four countries mark progress towards redressing some of the historical imbalances of that welfare model. In particular, fully-fledged minimum income schemes now operate in Portugal and in certain Spanish regions, while an experiment has been carried out involving a number of Italian municipalities. In view of this, the article concludes that social safety nets in southern Europe remain frail in terms of institutional design as well as political support and legitimacy.
\end{abstract}

\title{
INTRODUCCIÓN
}

La reforma del Estado del bienestar, quizá la más resistente y fructífera institución del pasado siglo, se mantiene como uno de los debates más controvertidos $\mathrm{y}$, en ocasiones, más ásperos de nuestros tiempos. Al tiempo que la discusión sobre el futuro del "modelo social europeo" cobra mayor interés, la atención principal de las discusiones suele concentrarse en el futuro de las pensiones y en otras políticas de considerable peso presupuestario, como la sanidad. Por su parte, la asistencia social, que concita el interés analítico de este artículo, permanece como un área de menor interés para los decisores públicos (policy makers), y ello a pesar de la mayor preocupación mostrada por las instancias comunitarias europeas en la lucha contra la pobreza y la exclusión social. Las fuerzas más activas en el proceso de reforma del bienestar, entendido éste como la gradual transición de un tipo de mercado laboral, de familia y de configuraciones de protección social a otro, han tomado conciencia de la importancia de la asistencia social y promocionan su mayor peso específico en el conjunto operativo del Estado del bienestar.

Las argumentaciones son bien conocidas: los fundamentos de la Época Dorada del capitalismo del bienestar de la segunda posguerra mundial han cambiado. En concreto, el fin del fordismo y el auge de la Nueva Economía han supuesto un duro golpe a la capacidad del mercado laboral como sostén del Estado del bienestar (Esping-Andersen, 2002). Los sistemas de protección social asumieron entonces que los mercados (laborales) asegurarían rentas estables a la mayoría de los asalariados, de tal manera que el Estado (del bienestar) se limitaría a proteger a los ciudadanos más mayores o más jóvenes que no podían ser activos en el mercado laboral, o a aquellos incapaces de incorporarse a la actividad laboral a causa de enfermedad o invalidez. Sin embargo, las expectativas generalizadas 
de empleos constantes a lo largo de la biografia laboral, a menudo en la misma empresa, han sido reemplazadas por una creciente inseguridad laboral, frecuentes cambios de empleo, periodos en el paro de larga duración, o un incremento del trabajo precario (Taylor-Gooby, 2001). En las situaciones en las que la protección social depende del estatus ocupacional, la inestabilidad del mercado laboral suele traducirse en pobreza y exclusión social (Ferrera et al., 2000).

Mientras el cambio económico socavó los cimientos del mercado laboral basado en el modelo del "varón sustentador" (male breadwinner model), el cambio social ha posibilitado que los encajes domésticos que los sustentaban fuesen cada vez menos prevalentes. Matrimonios a edades más avanzadas, menos hijos por pareja, creciente inestabilidad conyugal y otras manifestaciones de la "crisis de la familia" han minado la asunción tradicional de que los maridos asalariados mantenían al ama de casa y a sus dos o más descendientes. Las familias tradicionales a menudo actuaban como mecanismo redistributivo (puesta en común de recursos a favor de los miembros más necesitados), y como proveedor de servicios sociales (implicando a las mujeres en los cuidados sin remuneración a niños, mayores y enfermos). Dado que las familias en los últimos tiempos no han podido (y, quizás, no han querido) asumir tales funciones, las tensiones para los sistemas formales de protección se han hecho mayores (Lewis, 2001). Además, los cambios demográficos han evidenciado tales tensiones con demandas más apremiantes respecto a las prestaciones relativas a las pensiones, la atención sanitaria y a los cuidados sociales.

La multiplicación de carreras profesionales atípicas y de las nuevas formas familiares ponen en entredicho la capacidad de las configuraciones actuales del bienestar para el sostenimiento de rentas y para prevenir la pobreza. Al desaparecer hábitos y normas en los que se sustentaba la relación ocupacional de los asalariados y la relación familiar de las personas dependientes en los sistemas tradicionales de protección social, la existencia de "mallas de seguridad" bien diseñadas y eficaces cobra una importancia estratégica crucial en la lucha contra la pobreza y la exclusión. A la vista de tales tendencias, la asistencia social -el componente del Estado del bienestar mejor conformado para hacer frente a estos riesgos - adquiere una relevancia creciente (Saraceno, 2002; Moreno, 2003).

$\mathrm{La}$ asistencia social en la Europa del Sur ha sido descrita a menudo como rudimentaria (Gough, 1996; Leibfried, 1993). Tal apelativo no es del todo injustificado, ya que las mallas de seguridad meridionales carecen de algunos de los materiales más consistentes de sus homónimas del Norte de Europa. Sin embargo, los últimos desarrollos requieren de análisis más detallados al respecto.

Este artículo persigue contribuir a tales análisis mediante el examen crítico de las experiencias de las políticas contra la pobreza en España, Grecia, Italia y Portugal. En la siguiente sección se pasa revista a los condicionantes para establecer mallas de seguridad eficaces en la Europa del Sur. A continuación, se realiza una sintética descripción de las innovaciones llevadas a cabo en los cuatro 
RIS

REVISTA INTERNACIONAL DE SOCIOLOCIA

№ 36, Septiembre-Diciembre, 2003

LUIS MORENO, MANOS MATSAGANIS, MAURIZIO FERRERA y LUIS CAPUCHA

países meridionales europeos. Finalmente, el artículo concluye con una discusión acerca de las cuestiones pendientes y sus implicaciones para el futuro de estas políticas.

\section{LAS MALLAS Y SUS POLÍTICAS}

La cuestión del robustecimiento y la consolidación de las mallas de seguridad de protección social ha tenido una particular resonancia en la Europa del Sur. El papel marginal de la asistencia social, característico del régimen mediterráneo de bienestar (Ferrera, 1995; Sarasa \& Moreno, 1995; Rhodes, 1996), ha posibilitado que las estrategias de lucha contra la pobreza en estos cuatro países hayan sido limitadas. Según datos de 1996, las transferencias sociales (excluidas las pensiones) reducían de uno a tres puntos porcentuales los niveles de pobreza en Grecia e Italia, si bien su eficacia era superior - aunque por debajo del promedio en la UE- en España y Portugal.

Las modestas actuaciones en la lucha contra la pobreza caben ser atribuidas, al menos parcialmente, a la incapacidad para hacer llegar suficientes recursos a los pobres. En Grecia e Italia, donde el problema es más serio, sólo el $31 \%$ de las personas en el quintil más bajo de renta perciben prestaciones sociales, excluidas las pensiones (Marlier \& Cohen-Solal, 2000). De acuerdo con este dato, muchos hogares pobres no se pueden seleccionar para recibir asistencia social porque no cumplen con las condiciones establecidas en los diversos programas. Entre los afectados se encuentran los parados de larga duración (cuyas prestaciones y subsidios de desempleo se han extinguido), los trabajadores que se incorporan al mercado laboral (y que no pueden acceder a los subsidios contributivos), o los asalariados en situaciones laborales precarias, por citar algunos ejemplos.

Caer en situaciones de pobreza a través de los "agujeros" de la malla de seguridad es una experiencia usual entre los colectivos de inmigrantes y sus familias. Aunque los trabajadores extranjeros en situación de regularidad disponen de plenos derechos sociales, los inmigrantes ilegales sólo pueden acceder a los servicios de urgencia. Éstos son provistos en buena parte de los casos por organizaciones benéficas y/o por redes informales de apoyo dentro de sus colectivos de referencia (Baldwin-Edwards \& Arango, 1998). Entre otros factores, debe hacerse notar que la menor elegibilidad de los trabajadores inmigrantes es un efecto inevitable de un régimen del bienestar que, como el mediterráneo, se fundamenta en la ocupación formal, en el principio de la seguridad social y en el apoyo de la familia extensa. Desde esta perspectiva, el establecimiento de una malla de seguridad de carácter universal cobra una importancia adicional como factor instrumental para la emancipación de los excluidos y el mantenimiento de la cohesión social.

En cualquier caso, conviene señalar que el carácter fragmentario y "parcheado" de las mallas de seguridad en la Europa del Sur no es síntoma de un subdesa- 
Tabla 1.

Algunos indicadores de la situación social en la Europa del Sur.

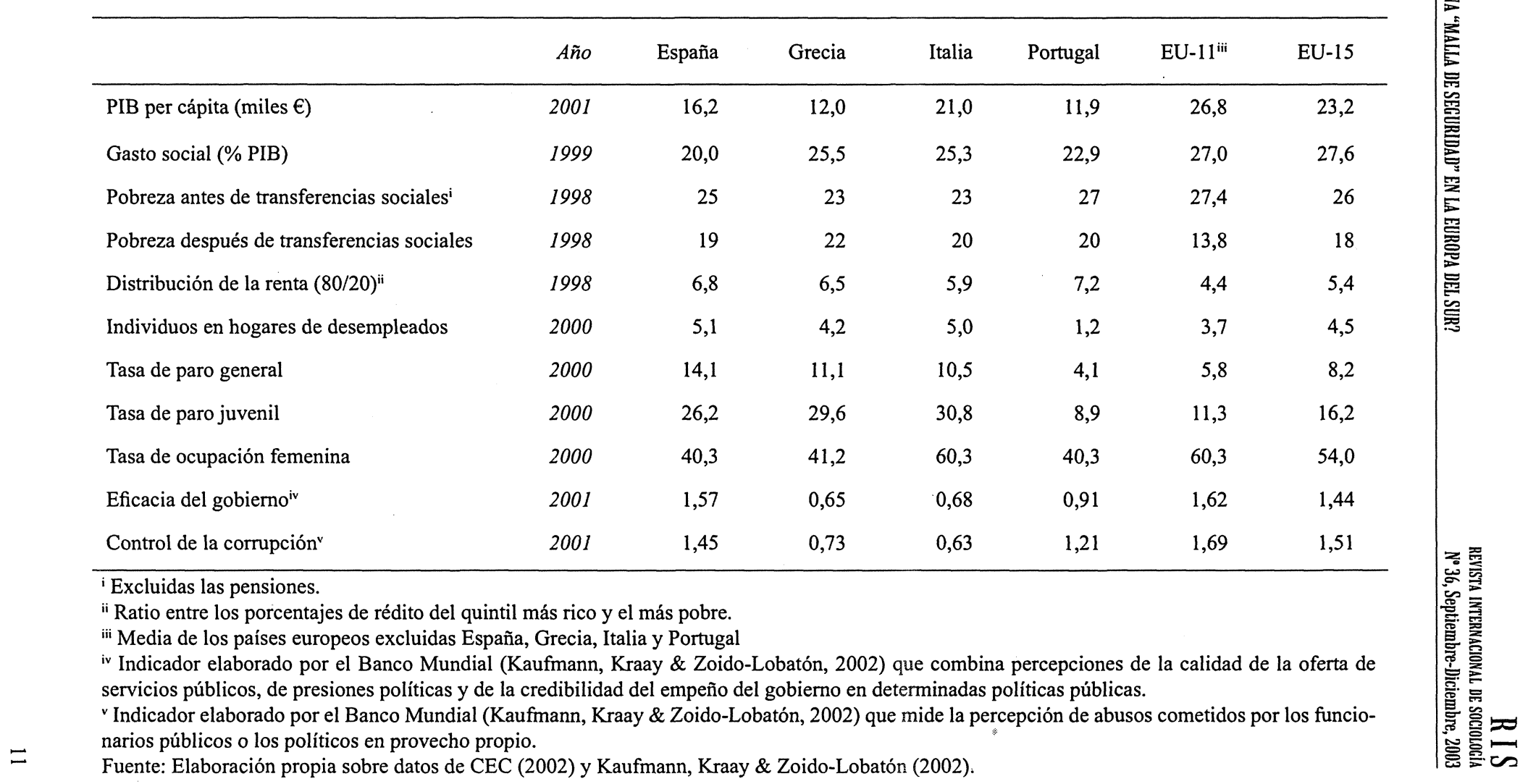


RIS

REVISTA INTERNACIONAL DE SOCIOLOGIA

№ 36, Septiembre-Diciembre, 2003

LUIS MORENO, MANOS MATSAGANIS, MAURIZIO FERRERA y LUIS CAPUCHA

rrollo de las instituciones del bienestar. Al contrario, el relativo desinterés por la implantación de una dimensión comprensiva por la lucha contra la pobreza ha coincidido a menudo con un crecimiento sostenido del gasto social. Algunos programas de alto gasto presupuestario (por ejemplo, las pensiones en Italia o Grecia) se han "sobredesarrollado" hasta el punto de hacer realmente difícil la inversión pública en otras políticas sociales. Consecuentemente, las causas del bajo perfil de la asistencia social en la Europa del Sur deben buscarse en el específico contexto de condicionantes y oportunidades que inhiben tal desarrollo. Dos de los condicionantes más relevantes están representados por el papel de la familia y la morosidad (softness) de sus instituciones estatales.

En los países de la Europa meridional las familias han funcionado tradicionalmente como eficaces (aunque informales) amortiguadores sociales y últimas redes de protección en una amplia gama de situaciones para la seguridad de los ciudadanos, tales como la atención y cuidados a niños y mayores, la ayuda a los desempleados, o la provisión de vivienda y de asistencia social con carácter general. Los datos demuestran que la "puesta en común de recursos" (resource pooling) y la compensación de riesgos en el ámbito familiar se ha intensificado en los últimos años (Fernández Cordón, 1997). Sin embargo, y a medida que la familia está siendo sometida a un mayor estrés, su capacidad para funcionar como una eficiente agencia de servicios domésticos es cada vez más incierta (Moreno, 2002). En realidad, el "familialismo" se basa fundamentalmente en el trabajo no remunerado de las amas de casa, pero tal disponibilidad femenina se hace cada vez más difícil de simultanear con una participación plena en el mercado laboral, lo que implica que un creciente número de mujeres se vean abocadas a carreras profesionales discontinuas o erráticas, o, simplemente, a renunciar a ellas. Salvo el caso de Portugal, los todavía bajos niveles de participación laboral femenina son indicativos de los altos costos sociales del régimen de bienestar mediterráneo (Saraceno, 2000).

Por otra parte, la provisión de prestaciones selectivas (targeted) requiere de un grado de eficiencia administrativa no siempre presente en las instancias gubernamentales públicas de la Europa meridional. En concreto, los sistemas administrativos sufren de una baja capacidad de implementación administrativa a causa de la persistencia de sistemas jerarquizados, y la falta de autonomía política para responder con diligencia a las necesidades expresadas en los niveles más próximos a los ciudadanos. El caso de la descentralización en España y el fuerte desarrollo de los mesogobiernos de las Comunidades Autónomas es la excepción a un contexto meridional generalmente caracterizado por una burocracia inercial. Tampoco se ven libres los proveedores públicos de recibir presiones externas a sus cometidos administrativos, o de disponer de insuficientes recursos para optimizar su labor. En base a ello, se generan en algunos territorios meridionales relaciones de mediación entre administradores públicos y beneficiarios (como ha podido ser el caso del Mezzogiorno italiano). Además, la fragmentación de buena parte 
de los programas de asistencia social favorece solapamientos normativos y de elegibilidad, al tiempo que deja a algunos colectivos desprovistos de una efectiva actuación administrativa.

Dado que las prestaciones de asistencia social se otorgan de acuerdo a la comprobación de recursos (means testing), la capacidad de los administradores para valorar con equidad y precisión el estado de necesidad de los potenciales beneficiarios es de crucial importancia. En este sentido, la Europa meridional representa un desafio para la política social: una variedad de factores como los hogares amplios, las altas tasas de trabajo tanto autónomo como sumergido, y la endémica evasión fiscal se combinan creando una situación peculiar. Uno de sus posibles efectos es la dificultad generada para los administradores a la hora de juzgar las circunstancias materiales de los solicitantes de las prestaciones sociales y su real elegibilidad para los programas disponibles (Atkinson, 1998).

De lo hasta aquí expuesto se deduce que la construcción de mallas de seguridad en un contexto propio del Sur de Europa caracterizado por pautas socioeconómicas complejas, una limitada capacidad administrativa y un persistente fraude fiscal, confronta dilemas específicamente meridionales (Addis, 1999; Aguilar et al., 1995). A consecuencia de ello, una transposición mecánica de conocimientos y experiencias desde los sistemas de asistencias social más experimentados de los países del Norte de Europa resultaría inadecuada para ofrecer respuestas ajustadas a las cuestiones reseñadas. Consiguientemente, a los decisores públicos (policy makers) en la Europa del Sur no les queda otra alternativa que explorar soluciones originales.

En buena medida, esto último es lo que ha ocurrido durante los últimos años. A pesar de las dificultades estructurales mencionadas, un énfasis renovado en las políticas de lucha contra la pobreza ha conducido a los países de la Europa meridional a introducir en sus sistemas de asistencia social un conjunto de políticas innovadoras. El emblema de este cambio de políticas (policy shift) ha sido la difusión de los programas de rentas mínimas, la ausencia de las cuales se había considerado como un rasgo característico del modelo meridional del bienestar (Gough, 1996). El éxito en Francia de la introducción del Revenue Minimum d'Insertion en 1988 desencadenó la adopción de similares programas en la Europa del Sur. Así sucedió en el País Vasco en 1988 con la introducción del Plan de Lucha contra la Pobreza, el cual sirvió a su vez de estímulo y referencia para los distintos programas de rentas mínimas de inserción en todas las Comunidades Autónomas españolas (Arriba, 2002). Un programa piloto fue implementado en Portugal en 1996, el cual pasó a ser plenamente efectivo con carácter nacional en 1997 (Capucha, 1998), mientras que en Italia tuvo lugar un programa experimental en diversos municipios durante 1998 y 2002 (IRS et al., 2001). Grecia ha sido el único país sin programas de rentas mínimas, aunque la cuestión del fortalecimiento de la malla de seguridad ha ganado relevancia en el debate público (Matsaganis et al., 2001). Las trayectorias de tales políticas en los contextos de 
los cuatro países meridionales de la Unión Europea constituyen a continuación el objeto de nuestro análisis.

\section{PERFILES NACIONALES}

\section{España}

Durante el franquismo los programas de asistencia social eran muy escasos. La Iglesia y la familia eran los principales proveedores de bienestar a las personas necesitadas. La Constitución democrática de 1978 inauguró un período de institucionalización de la asistencia y servicios sociales. De acuerdo con las provisiones constitucionales, la legislación básica y la seguridad social permanecieron bajo competencia del Estado central, mientras que la asistencia social pasaba a ser una competencia "exclusiva" de las 17 Comunidades Autónomas. Éstas reclamaron servicios y competencias en sus estatutos de autonomía. Entre 1982-93, y tras la promulgación de leyes autonómicas, se establecieron sistemas regionales de servicios sociales de acceso universal a los ciudadanos.

En 1987, un acuerdo entre los gobiernos central, autonómicos y locales posibilitó el Plan Concertado para el Desarrollo de Prestaciones Básicas de Servicios Sociales de las Corporaciones, el cual articuló la cooperación administrativa entre los tres niveles gubernamentales en la provisión de red de servicios primarios y de asistencia social. En 1988, y con efectos desde 1990, se generalizaron las pensiones no contributivas de jubilación, invalidez y prestaciones por hijo a cargo, las cuales son un componente esencial de la malla de seguridad en España. Tales prestaciones no contributivas son asignadas tras la comprobación de recursos (means testing) de los peticionarios mayores de 65 años, las personas con un grado del $65 \%$ o más de invalidez, y las familias necesitadas con hijos a su cargo. Con antelación, y en 1984, se habían implementado subsidios por desempleo para aquellos parados que hubiesen finalizado su seguro de paro sin haber encontrado empleo.

Por su parte, todas las Comunidades Autónomas pusieron en marcha los programas de rentas mínimas de inserción. Como se ha indicado, el primero se introdujo en el País Vasco en 1989 siguiendo las líneas maestras del RMI francés. El último comenzó a funcionar en las Islas Baleares en 1995. Los programas autonómicos de rentas mínimas comparten un mismo propósito general, aunque difieren en su alcance, cobertura o fórmulas de inserción de los beneficiarios, un aspecto sobre el cual retornaremos más adelante. El gasto público involucrado en el mantenimiento de estos programas es pequeño (apenas un $0,3 \%$ en promedio de los presupuestos autonómicos consolidados de 2000). Su importancia, empero, estriba principalmente en dos aspectos: (a) Son programas innovadores, al haber sido diseñados y puestos en vigor por las propias Comunidades Autónomas; y 
(b) se trata de actuaciones que 'cierran' la malla de seguridad al poder acceder personas no elegibles en otros programas de subsidios sociales (de acuerdo con estimaciones del Ministerio de Trabajo y Asuntos Sociales, los beneficiarios individuales superaron los doscientos mil en el año 2000) (Arriba \& Moreno, 2002).

En abril de 1995, la Ponencia para el Análisis de los Problemas Estructurales del Sistema de la Seguridad Social y de las Principales Reformas que deberán acometerse, fue aprobada en el Congreso de los Diputados con el apoyo explícito de los partidos y sindicatos más representativos. Lo que posteriormente fue conocido como "Pacto de Toledo", auspició un gran acuerdo social y un posterior desarrollo legislativo y político de amplias repercusiones para el sistema de protección social en España. Entre sus objetivos conviene resaltar la financiación diferenciada de las prestaciones contributivas y no contributivas. Como consecuencia de ello, la atención universal sanitaria y los servicios sociales, así como las pensiones no contributivas y las prestaciones de asistencia social por comprobación de recursos, serían financiadas a través de la fiscalidad general, acentuando, así, su carácter progresivo y redistributivo. Las prestaciones contributivas se realizarían con cargo al sistema de la seguridad social y se crearía un Fondo de Reserva con aportaciones de los superávit, el cual alcanzó la cifra de 8 mil millones de euros en julio de 2003 (Rodríguez-Cabrero, Arriba y Marbán, 2003). Reformas recientes han insistido en la "activación" laboral de los peticionarios, en la adopción de criterios más estrictos de acceso a los subsidios asistenciales de desempleo, y en el establecimiento de mínimos vitales y ayudas familiares en forma de desgravaciones y exenciones fiscales. Las principales prestaciones económicas asistenciales en España son analizadas sintéticamente como sigue:

a) Prestaciones suplementarias de la seguridad social. Los complementos de mínimos de pensiones de la seguridad social elevan las cantidades a percibir hasta un mínimo legalmente establecido, y alcanzan a un $30 \%$ de todas las pensiones contributivas retribuidas a 2.400 .000 beneficiarios en el año 2000. Por su parte, los subsidios de desempleo (incluido el agrario) y la renta activa de inserción laboral, se otorgan, previa la comprobación de recursos, a unos 600.000 trabajadores en paro sin acceso a las prestaciones de paro de la seguridad social.

b) Prestaciones no contributivas de la seguridad social. Incluyen las pensiones no contributivas de jubilación y las de invalidez, así como las pensiones asistenciales y los subsidios de garantía de mínimos (LISMI). El acceso a estas prestaciones se realiza tras la comprobación de recursos de los peticionarios sin un historial completo en el sistema de la seguridad social. Disfrutaban de las mismas alrededor de 620.000 personas en 2000. En segundo lugar, dentro de este apartado, están las prestaciones familiares de la seguridad social destinadas a las familias necesitadas con hijos a cargo. Otras prestaciones se pusieron en vigor en 2000 por el nacimiento del tercer y sucesivos hijos, y en la eventualidad de partos múltiples. 
RIS

REVISTA INTERNACIONAL DE SOCIOLOCIA

$N^{\circ} 36$, Septiembre-Diciembre, 2003

LUIS MORENO, MANOS MATSAGANIS, MAURIZIO FERRERA Y LUIS CAPUCHA

c) Las rentas minimas de inserción son programas no contributivos sujetos a comprobación de recursos y de responsabilidad de las Comunidades Autónomas. Constituyen la última instancia disponible las prestaciones de carácter asistencial. En 2000, unas 80.000 familias se han beneficiado de estos programas, lo que supone aproximadamente un $0,5 \%$ de la población total española.

Tabla 2.

Programas de rentas minimas (2000).

\begin{tabular}{lcccc}
\hline & España & Grecia & Italia & Portugal \\
\hline Renta mínima por individuo & $286^{\mathrm{a}}$ & $148^{\mathrm{b}}$ & 268 & 125 \\
( mensuales) & $386^{\mathrm{a}}$ & 444 & 660 & 374 \\
Renta mínima por pareja y 2 hijos & 202 & 700 & $86^{\mathrm{c}}$ & 418 \\
Número de beneficiarios (miles) & 0,5 & 6,4 & $3,6^{\mathrm{c}}$ & 4,2 \\
Número de beneficiarios (\% población) & 210 & 269 & $110^{\mathrm{d}}$ & 284 \\
Coste del programa (millones $€$ anuales) & 0,03 & 0,23 & $0,22^{e}$ & 0,25 \\
Coste del programa (\% PIB) & & & &
\end{tabular}

${ }^{a}$ Importe de la prestación en Cataluña. Las restantes cifras en el caso de España se refieren al conjunto de los programas autonómicos de rentas mínimas.

b Todas las cifras en el caso de Grecia, donde no existe ningún programa de renta mínima, son estimaciones resultado de un ejercicio de simulación (efectuado en Matsaganis et al. 2001).

${ }^{c}$ El número de beneficiarios (y el porcentaje de la población local involucrada) en los 39 municipios que participaron en la primera fase de experimentación del experimento de reddito minimo d'inserimento.

d Mitad del coste del programa en los 39 municipios experimentales durante el período de dos años (1999-2000).

e Estimaciones realizadas en 2001 (\% PIB) si el programa se hubiera generalizado en toda Italia.

Fuente: Informe final FIPOSC.

Los programas de rentas mínimas difieren respecto a su alcance, cobertura o fórmulas de inserción de los beneficiarios. Sólo el correspondiente al País Vasco puede considerarse como un genuino programa de rentas mínimas garantizadas. Otros programas bien desarrollados y generosos en cuanto a sus prestaciones son los correspondientes a Madrid, Cataluña o Navarra. Por su parte, otras regiones disponen de programas de una cobertura limitada y un nivel de prestación menor, u ofrecen empleo temporal en proyectos de utilidad social (Aguilar et al., 1995). 
En 2000, la cantidad básica mensual para los beneficiarios que vivían solos variaba desde 239 euros, en las Islas Canarias, a 305 euros, en el País Vasco y 319 euros en Extremadura y Navarra (por comparación, considérese que el salario mínimo era de 496 euros y las pensiones no contributivas de 288 euros, ambas cantidades mensuales). El ajuste de los subsidios de acuerdo al tamaño de los hogares se realiza de acuerdo a escalas de equivalencia (la presencia de un miembro adicional puede incrementar el total del subsidio concedido hasta un 30\% del nivel básico), aunque la cantidad final de la prestación está sujeta a un límite máximo. El gasto total agregado de las rentas mínimas de inserción autonómicas alcanzó la cifra de 210 millones de euros (0,03\% del PIB). En el contexto de los presupuestos autonómicos, el gasto de los programas de rentas mínimas de inserción ha fluctuado desde los 337,000 euros de La Rioja (por debajo del $0,1 \%$ del presupuesto autonómico), hasta los 53 millones de euros en el País Vasco (por encima del 1\%). Andalucía, Cataluña y Madrid han gastado en estos programas 89 millones de euros entre las tres (Arriba y Moreno, 2002).

Las desarrollos futuros de los programas autonómicos de rentas mínimas de inserción son hasta cierto punto inciertos. Su implementación contribuyó a la legitimación de las Comunidades Autónomas y se vio favorecido por el crecimiento de los recursos presupuestarios. Nótese que de un 3\%, en 1981, el gasto autonómico creció hasta un 33\% del total del gasto público, en 2001. Las nacionalidades y regiones españolas han sido capaces de integrar efectivamente asistencia y servicios sociales en una red primaria local, aunque buena parte de los programas sufren problemas de financiación. Además, su naturaleza descentralizada podría exacerbar las diferencias regionales, algo que hasta el momento no ha sucedido. En cualquier caso, una armonización a nivel estatal de los programas llevada a cabo de manera jerárquica no aparece como una opción viable en un país federalizado como es España (Moreno, 1999). Quizá en un futuro no lejano las Comunidades Autónomas se vean abocadas a requerir la cofinanciación desde el gobierno central, a mantener los programas, o a revisar el desarrollo ulterior de los mismos.

El Plan Nacional de Acción para la Inclusión Social del Reino de España de 2001-03 ha constituido una buena oportunidad para la coordinación intergubernamental, ofreciendo un primer cuadro sintético de la situación de la lucha contra la pobreza en España. Resta por comprobar si las próximas acciones y puestas en común en este proceso auspiciado por la UE serán fuentes de nuevas ideas (e, idealmente, de fondos) para optimizar los esfuerzos de todos los actores e instituciones implicados.

\section{Grecia}

La restauración de la democracia en 1974 propició un período de expansión del Estado del bienestar griego, acelerada después de la victoria del partido socialista 
en las elecciones generales de 1981. El incremento sin precedentes del gasto social fue una respuesta a las expectativas forjadas por amplios sectores de la población durante décadas de discriminación política. La entrada de Grecia en la Comunidad Europea en 1980, ampliamente considerada como una garantía de estabilidad política, legitimó sus aspiraciones a niveles de renta y protección social comparables a las de otros europeos (Guillén y Matsaganis, 2000).

Actualmente, un elevado y creciente nivel de gasto social, que se ha aproximado rápidamente a la media europea, se combina con una débil actuación respecto a las transferencias sociales para reducir la pobreza. Esta aparente contradicción cabe ser atribuida a la naturaleza del sistema de protección social griego. El sistema contributivo de la seguridad social encaja bien en un contexto "fordista" de carreras laborales largas e ininterrumpidas. Por la misma razón, los parados de larga duración, las mujeres con una irregular biografia laboral, los trabajadores con empleos temporales o a tiempo parcial, los inmigrantes ilegales, los que permanecen en la economía "sumergida" y otros colectivos son marginados u outsiders de la seguridad social, para los cuales el bienestar queda seriamente reducido con harta frecuencia.

En realidad, el Estado del bienestar griego pone el énfasis en las prestaciones contributivas, con una provisión pequeña para la cobertura de los riesgos sociales no asegurativos, tales como la pobreza, mientras que los servicios sociales permanecen en un estadio inicial de desarrollo. Consecuentemente, mientras las pensiones suponen la mayor parte de las transferencias sociales, las políticas dirigidas a las familias con hijos, los discapacitados, los parados y otros grupos bajo el riesgo de la pobreza se han desarrollado en menor medida.

En general, la asistencia social ha permanecido en una posición marginal y sus prestaciones no se han integrado adecuadamente, siendo administradas por diversos organismos y bajo diferentes normativas. A resultas de esta interacción existe una estructura irregular, carente de coherencia en su conjunto y que combina solapamientos en la elegibilidad con lagunas en la cobertura. Dado que las transferencias no contributivas están mejor conformadas para la lucha contra pobreza, la índole marginal de la asistencia social en Grecia deja una malla de seguridad llena de agujeros, a través de los cuales los individuos y sus familias pueden caer en situaciones de pobreza. Los hogares son inelegibles para los programas sociales existentes si no se ajustan al "perfil" imaginado por los legisladores, ni cumplen los estrictos requisitos establecidos.

La malla de seguridad para los ciudadanos mayores es también fragmentaria. Aquellos con un historial de vida laboral contributiva suficiente disponen de una pensión mínima, además de un complemento previa comprobación de recursos. Los campesinos y, en general, las personas con una baja renta y sin otros derechos, pueden recibir pensiones no contributivas. No existe un programa de ingresos mínimos garantizados en Grecia, lo que genera una situación insólita con una combinación de un gasto social elevado en pensiones (13\% del PIB) y, al mismo 
tiempo, un alto porcentaje de viejos pobres ( $35 \%$ de los mayores de 65 años) (Matsaganis, 2002).

La fragmentación y una cobertura incompleta son evidentes en todas las otras áreas del sistema de seguridad social. El seguro de paro es de carácter contributivo y por un período de tiempo limitado (12 meses), a resultas de lo cual sólo el $44 \%$ de los inscritos como desempleados recibieron la prestación en 1999. Las transferencias de rentas a las familias están dirigidas sólo a aquéllas con tres o más hijos, lo que provoca que los niños pobres de familias más reducidas no reciban ayuda o la reciban muy escasamente. Las prestaciones por invalidez varían según el grado de invalidez y el estado (existen 10 categorías y 22 subcategorías de subsidios). Las ayudas a la vivienda son de índole contributiva y están encaminadas a facilitar la propiedad de la casa, con lo cual están fuera del alcance de las familias pobres.

En 2001, el conjunto las prestaciones no contributivas sumaron el $16,3 \%$ de todo el gasto de la seguridad social, mientras que aquéllas sujetas a comprobación de rentas alcanzaron un magro 4,7\%. La gradual desaparición de las pensiones básicas para los campesinos desde 1998, y la abolición de la prueba de comprobación de rentas en el caso de los subsidios por "muchos hijos" en 2002 (9,3 y 1,9\%, respectivamente, del gasto total de la seguridad social), reducirán aún más el espacio reservado a estos dos tipos de subsidios en el sistema de protección social griego.

Desde 1996, el criterio de selectividad (targeting) se ha extendido a partir de que el gobierno socialista, bajo un liderazgo nuevo, declarase como objetivo prioritario la pertenencia de Grecia a la Unión Económica y Monetaria con un compromiso en pos de una "sociedad cohesionada". El concepto de selectividad fue adoptado como una forma de "cuadrar el círculo" de la protección social. La consiguiente estrategia puesta en marcha dio sus primeros frutos (el complemento de las pensiones previa comprobación de recursos mencionada líneas arriba), pero perdió "fuelle" presuntamente por la ausencia de grupos seleccionados con nitidez, dado que el sistema ha continuado dominado por las prestaciones de carácter contributivo.

El Plan de Acción Nacional para la Inclusión Social descartó la opción del programa de rentas mínimas al tiempo que reiteraba su empeño por políticas selectivas. Se anunciaron para 2002 la puesta en vigor de tres nuevas actuaciones, la más prometedora de las cuales es la ayuda asistencial de desempleo para parados de larga duración que sean miembros de familias de renta baja, con edades comprendidas entre 45 y 65 años, y a percibir por un período de 12 meses. Sin embargo, diez meses después de la implementación del programa sólo 711 de los 35.000 desempleados inscritos, y con capacidad para solicitar la prestación, lo habían hecho. El bajo porcentaje de adhesión al programa afectaba igualmente a otro ambicioso programa, implementado en 2000 , y relativo a los descuentos de cotizaciones sociales para trabajadores retribuidos con el salario mínimo.

En términos generales, el peligro de que algunos grupos en situaciones de pobreza no sean elegibles para programas de ayuda de rentas (CEC, 2001) no 
RIS

REVISTA INTERNACIONAL DE SOCIOLOCIA

№36, Septiembre-Diciembre, 2003

LUIS MORENO, MANOS MATSAGANIS, MAURIZIO FERRERA y LUIS CAPUCHA

ha disminuido sustancialmente a pesar de los avances realizados. La ausencia de una prestación de última instancia, de índole selectiva pero de alcance universal, es un elemento de encaje que falta en la malla de seguridad griega. La oposición a la introducción de un programa de rentas mínimas deja al tejido de la red de protección social en una situación de fragilidad. Aunque no se deben menospreciar las dificultades administrativas implicadas en la implementación de un programa de tal calibre, las necesidades de financiación serían bastante modestas: según un ejercicio de simulación el coste de las transferencias de un programa nacional de rentas mínimas era de 269 millones de euros, o 0,23\% del PIB, en 2000 (Matsaganis, et al., 2001).

\section{Italia}

La pobreza y la exclusión social, temas marginales en la agenda y debate políticos italianos, han concitado un creciente interés en la segunda mitad de los años noventa. Un diagnóstico de la assistenza ha sido compartido por actores políticos y agentes sociales indicando sus principales defectos: alta fragmentación, solapamientos programáticos, una preferencia por las transferencias monetarias en vez de los servicios, una marcada diferenciación territorial y la ausencia de una malla de seguridad como "última red" de protección social.

En 1977 la competencia de la asistencia social fue descentralizada a los gobiernos regional y local, aunque la definición de los principios rectores y los estándares nacionales permanecieron como responsabilidad de la administración central y debían ser reguladas por una ley marco. Como dicha norma general no se promulgó hasta el año 2000, en el ínterin las leyes regionales permitieron una gran discrecionalidad a nivel municipal. Por ello, algunos programas de rentas mínimas de carácter local fueron puestos en vigor a iniciativa de los municipios y ciudades. Turín, en 1978, Ancona, en 1981, Catania, en 1983 y Milán, en 1989, introdujeron programas con una prestación no categórica sujeta a comprobación de recursos y conocida como minimo vitale. Muchos otros municipios y ciudades (como por ejemplo Bari o Roma) no llevaron a efecto tal iniciativa.

A nivel nacional, la asistencia social atiende a categorías específicas, tales como la vejez y la incapacidad, pero su papel es bastante modesto $(6,4 \%$ del total de gasto social en 2000). Las actuaciones de asistencia social incluyen las pensiones de invalidez civil (pensione di inabilità civile, assegno di assistenza), las cuales son prestaciones no contributivas, compatibles con la actividad laboral y sujetas a una estricta comprobación de rentas del beneficiario. La pensione sociale es un subsidio mínimo, concedido previa comprobación de recursos, para aquellos ciudadanos mayores de 65 años que no han madurado su derecho a una pensión contributiva. Tras la reforma Dini de 1995, las personas afectadas por el nuevo régimen podían ser elegibles para el assegno sociale (una prestación no contributiva para aquellas personas con recursos inadecuados o con contribuciones insu- 
ficientes). El subsidio de cuidados (indennità di accompagnamento) está dirigido a aquellas personas que necesitan atención permanente al no ser autónomos. Es condicional a la realización de una prueba médica pero no a la comprobación de recursos. Con el paso del tiempo se ha consolidado como fuente de ayuda a los colectivos más débiles de ancianos.

En general, los grupos de rentas bajas son potencialmente elegibles para diversos subsidios sociales contributivos. El subsidio familiar (assegno per il nucleo familiare) es una prestación sujeta a la comprobación de recursos para asalariados activos o jubilados con cargas familiares. El nivel del subsidio está directamente relacionado con las dimensiones de la familia y es inversamente proporcional a la renta familiar. Los complementos de pensiones (integrazioni al minimo) se abonan a quienes perciben prestaciones por debajo del mínimo legalmente establecido. Dichos complementos requieren de un biografía laboral contributiva de, al menos, 15 años y son sujetos a la comprobación de recursos de los solicitantes. Las personas que se han incorporado al mercado laboral después de 1995 no pueden solicitar los integrazioni al minimo, pero pueden acceder al nuevo assegno sociale antes mencionado. Las pensiones de invalidez (pensione di inabilità) requieren de un biografía laboral de los solicitantes de, al menos, 5 años. En 1984 los criterios de control médico se endurecieron y han pasado a realizarse periódicamente. Hasta esa fecha las pensiones de invalidez habían operado de facto como rentas mínimas, en particular en el Mezzogiorno, donde habían sido moneda de cambio para las relaciones clientelares entre políticos y electores (Ferrera, 1996). Considérese que en 1998 más de 3 millones de personas recibían pensiones de invalidez, de las cuales casi el $90 \%$ las venían percibiendo desde antes de la reforma de 1984 (Benassi, 2001).

La concentración de recursos en actuaciones de índole contributiva y/o categórica generaba huecos en la malla de seguridad para las personas de rentas bajas. Tal situación empezó a cambiar hacia mitad de los años noventa, de acuerdo a las recomendaciones de varias comisiones de expertos para combatir la pobreza y la exclusión social. En concreto, el Informe Onofri (1997) propuso la fórmula sobre la cual se han basado las sucesivas reformas de la asistencia social. En síntesis, el Informe enfatizaba la necesidad de promulgar una ley nacional de asistencia social y de introducir un programa de rentas mínimas no categórico. Recomendaba, igualmente, la eliminación gradual de algunos programas asistenciales, y el establecimiento de un nuevo mecanismo para determinar la situación económica de los peticionarios.

Algunas innovaciones significativas se llevaron a cabo tras la publicación del Informe Onofri. Un nuevo conjunto de reglas para establecer y valorar el estado de necesidad de los potenciales beneficiarios fue introducido en 1998 (Indicatore della situazione economica, ISE). Tal indicador especifica cómo la renta y los bienes del peticionario deben ser considerados cuando se evalúa una solicitud sujeta a la comprobación de recursos. Entre otros aspectos, el ISE trata de corregir los 
R I S

REVISTA INTERNACIONAL DE SOCIOLOGIA

№ 36, Septiembre-Diciembre, 2003

LUIS MORENO, MANOS MATSAGANIS, MAURIZIO FERRERA y LUIS CAPUCHA

errores generados por la opacidad fiscal por parte de algunos tipos de potenciales beneficiarios (Baldini et al., 2002). El Indicatore se aplica a dos prestaciones introducidas en 1998: el subsidio para las familias numerosas (para aquéllas con 3 o más hijos), y el subsidio de maternidad (disponible para las que no tienen acceso al subsidio de maternidad contributivo). Además, en el año 2000 una nueva ley marco (328/2000) reformó el contexto institucional de la asistencia social italiana, estableciendo líneas generales de actuación y principios de descentralización y subsidiariedad, así como estándares para las actuaciones administrativas.

El experimento de las rentas mínimas dio comienzo en 1998. El Reddito minimo di inserimento (RMI) incluye una componente monetaria y otra de activación: el derecho a la ayuda económica es condicional a la participación en programas de inserción laboral y social. El nivel de la ayuda económica queda determinado por la diferencia entre el ingreso mínimo garantizado - ajustado a las dimensiones de la familia - y los recursos de que dispone el beneficiario (de los cuales quedan excluidos los posibles subsidios familiares percibidos, el $25 \%$ de las rentas de trabajo, los gastos médicos y, parcialmente, el alquiler de la vivienda). La mayor parte de los beneficiarios (93\% en el período 1998-2000) residían en el Sur, donde algunos municipios desarrollaron ingeniosos mecanismos adicionales para determinar la situación económica de los peticionarios, dadas las implicaciones de la economía sumergida en aquella zona. Durante 1998-2000, la primera fase del experimento de las rentas mínimas implicó a 34.700 familias en 39 municipios. La ley presupuestaria de 2001 prorrogó el programa piloto por otros dos años y elevó el número de municipios elegibles hasta 306. Sin embargo, a fines de 2003 el gobierno se disponía a no prorrogar los fondos para la continuación del experimento RMI.

El informe de evaluación de la primera fase del programa piloto efectuado por tres institutos de investigación independientes ha arrojado luz sobre varios aspectos del programa (IRS, Fondazione Zancan y CLES 2001). Entre los aspectos positivos, el RMI —el componente ausente en la asistencia social italiana - rompe con una larga tradición de actuaciones categóricas y discrecionales de ayudas a las personas con baja renta, las cuales han demostrado su ineficacia como políticas contra la pobreza. Antes de 1998, los residentes en algunos municipios incluidos en el programa experimental no tenían acceso a ninguna forma de ayuda económica pública, aun en el caso de aguda situación de necesidad. Además de su primaria función como sostén económico, el experimento del RMI ha auspiciado innovaciones interesantes en el terreno de la activación de algunos beneficiarios, mediante la puesta en marcha de nuevos trayectorias de inserción y revitalización social (incluidas las medidas contra el abandono escolar). En algunos casos, también se ha facilitado la optimización administrativa con la asunción de nuevas responsabilidades directas en la gestión del programa.

Respecto a los aspectos menos sobresalientes, la evaluación ha evidenciado algunas críticas tales como la dificultad de resistir a las presiones de los contextos 
locales, de seleccionar correctamente a los beneficiarios, o de dotar al programa de un carácter diferente al benéfico-asistencial del pasado. La legislación del programa piloto preveía la posibilidad de generalizar el programa a todo el territorio nacional, aunque la decisión de no prorrogar presupuestariamente el experimento del RMI arroja serias dudas respecto a su viabilidad futura. El informe de evaluación realizaba una estimación del coste del RMI, a nivel nacional, de entre 2.2 y 3 mil millones de euros $(0,18 \%$ y $0,24 \%$ of GDP) en 2001 . Tal magnitud era significativa pero en modo alguno podía considerarse como excesiva comparada con otras políticas de bienestar.

Dos obstáculos parecen bloquear la completa implantación en Italia del programa de rentas mínimas: las débiles capacidades administrativas locales y las peculiares condiciones socioeconómicas del sur italiano. Como sucede con otros programas similares, el RMI es exigente en cuanto a capacidades institucionales y habilidades gerenciales. Además, existe un riesgo de sobrecarga funcional: en vez de un programa de última instancia, el RMI corre el peligro de representar la única opción disponible para obtener un mínimo de recursos en caso de necesidad.

De lo expuesto se colige que una mejora sustancial de las capacidades organizativas, a ambos niveles macro y micro, debe conllevar un mayor esfuerzo por reequilibrar las políticas laborales y las sociales familiares. La localización institucional más apropiada para el RMI debería ser en el nivel básico de los programas de transferencias monetarias a los desempleados y a las familias con hijos $\mathrm{u}$ otros dependientes a cargo. De la misma manera, el correcto funcionamiento de su función de inserción requiere de un sistema articulado de políticas activas para el mercado de trabajo y de servicios sociales de apoyo a las familias. Algunos ecos de esta diagnosis se han hecho sentir en el Plan de Acción Nacional para la Inclusión Social de 2001, a pesar de que éste plan no ilustra de manera clara cómo el programa del RMI debería ser rediseñado en sus objetivos concretos.

Como ya hemos indicado, el actual gobierno de centro-derecha no se ha mostrado dispuesto a la generalización en toda Italia del programa del RMI. Se ha dejado que las regiones decidan sobre el asunto, absteniéndose el gobierno Berlusconi de establecer estándares nacionales o de comprometerse a facilitar recursos financieros. El Pacto de Italia, auspiciado en el verano de 2002, preveía la implantación de una "renta de última instancia" como parte importante de una más amplia reforma del sistema de amortizadores sociales. Transcurrido un año no estaba del todo claro si tal proyecto se llevaría finalmente a cabo.

\section{Portugal}

Portugal se integró en la Comunidad Europea en 1986 poniendo fin a un largo período de atrasos iniciado 58 años antes con la dictadura del Estado Novo. Hasta aquella fecha no existían ni cobertura social universal ni sistema público sanitario 
RIS

REVISTA INTERNACIONAL DE SOCIOLOGIA

№ 36, Septiembre-Diciembre, 2003

LUIS MORENO, MANOS MATSAGANIS, MAURIZIO FERRERA y LUIS CAPUCHA

(Viegas y Costa, 2000). Durante la dictadura, la lucha contra la pobreza, que alcanzaba a un $40 \%$ de la población, no fue considerada como una prioridad política. La revolución democrática del 25 de abril de 1974 promovió la implantación de un conjunto de derechos e instituciones sociales que configuran un Estado del bienestar moderno. Sin embargo, las condiciones adversas en el período subsiguiente limitaron los recursos financieros e institucionales necesarios para poner en marcha las nuevas políticas sociales. En consecuencia, las actuaciones para combatir la pobreza fueron escasas y la asistencia social permaneció fragmentada.

En 1984 se reorganizó la protección social. Una ley marco estableció las bases de la seguridad social con una estructura a tres niveles, según las previsiones constitucionales. El régimen general proporciona las prestaciones contributivas a los trabajadores y sus familiares a cargo, mientras que el régimen no contributivo y la asistencia social se ocupan de las intervenciones no incluidas en el régimen contributivo. Las políticas contra la pobreza recibieron un gran impulso con la participación de Portugal en el II Programa Europeo contra la pobreza, el cual favorecía los proyectos de intervención y de investigación orientados a grupos específicos. Su énfasis en la participación facilitó su adaptación a la metodología de desarrollo local empleada entonces. Posteriormente, la política contra la pobreza se apoyó en mayor medida en un enfoque territorial integrado y en partenariados (partnerships) a nivel local y nacional (Almeida et al., 1994).

En 2000, cuando la legislación sobre la seguridad social fue revisada, el nivel y la composición del gasto social portugués se aproximaba a la media europea. La nueva ley tenía como objetivo elevar las prestaciones y asegurar la sostenibilidad de la seguridad social, mediante el reforzamiento del fondo para las pensiones públicas y la imputación en el presupuesto nacional de los gastos de la asistencia social. La reforma se sustentó en un acuerdo de los agentes sociales con excepción de la patronal CIP. La ley convalidó a una nueva generación de políticas sociales iniciada en 1995, las cuales pretendían activar no sólo a los individuos sino también a las instituciones a través de un enfoque individualizado y orientado a las condiciones de los ciudadanos y sus necesidades.

Indudablemente, el programa de las rentas mínimas (Rendimento Minimo Garantido, RMG) se convirtió en el emblema de la nueva generación de políticas sociales (Capucha, 1998). Durante el período que precedió a las elecciones generales de 1995, se produjo un debate de alto nivel sobre la recomendación 92/441 del Consejo Europeo de recursos suficientes (auspiciada durante la presidencia portuguesa de la UE), lo que puso el tema de la pobreza en un lugar prominente de la agenda política. El gobierno socialista que se formó tras las elecciones introdujo experimentalmente el RMG en 1996, y extendió su cobertura nacional a partir del 1 de julio de 1997.

El modo más simple de describir al RMG es que se trata de un contrato: facilita una ayuda monetaria como prestación no contributiva, a fin de garantizar un estándar mínimo de vida, a cambio de un compromiso por participar en un programa 
de inserción o integración social. El carácter innovador del RMG está en el hecho de que la oferta de asistencia económica se combina con la participación en una serie de actividades de inserción laboral, educativa, de formación profesional, de acceso a los servicios sanitarios, de actuaciones respecto a la vivienda, etc. Al programa pueden acceder todos los residentes legales en situación de agudo y demostrable estado de necesidad. La edad mínima de los beneficiarios es de 18 años, excepto padres, mujeres embarazadas o cuidadores de personas ancianas con discapacidades, de edad más joven.

El RMG no comporta la percepción de una cantidad fija sino la diferencia monetaria entre la renta neta evaluada del beneficiario y la cantidad garantizada para cada tipo de hogar. Esta última es igual a la pensión social para cada uno de los dos primeros adultos del hogar con incrementos del $70 \%$ de cada miembro adulto adicional y del $50 \%$ por cada hijo a cargo. En el cálculo de los recursos del hogar no se toman en consideración los subsidios familiares ni las becas de estudios que los miembros pudieran percibir. Además, y para favorecer la inserción laboral, el $20 \%$ de las rentas laborales o por formación tampoco se toman en cuenta (porcentaje aumentado al 50\% durante el primer año). Al ser el RMG subsidiario de otros subsidios sociales, los perceptores deben solicitar con carácter previo cualquier otra prestación a la que pudieran tener derecho. La renta mínima portuguesa se otorga por un año con prórrogas posteriores si los beneficiarios mantienen los requisitos iniciales. Los trabajadores sociales de los servicios sociales municipales evalúan las condiciones materiales de los potenciales beneficiarios. En la eventualidad de diferencias sustanciales entre la renta declarada y la real percibida el RMG queda ajustado o incluso cancelado.

El trabajador social a cargo de la solicitud del RMG emite un informe individual que debe incluir una descripción de los problemas confrontados por el hogar, y propone un plan de inserción. Se trata de un acuerdo firmado entre el trabajador social, en representación del comité local de supervisión, y los miembros de hogar. $\mathrm{El}$ acuerdo especifica las tareas a cumplir por los beneficiarios y la ayuda otorgada por las instituciones locales. En el supuesto que el acuerdo resultase inadecuado, o requiriese de álguna modificación, puede ser renegociado sucesivamente con el beneficiario. El disfrute de la prestación es condicional a la participación en el plan de inserción, y todo el proceso posee efectos legalmente vinculantes.

A fines de 2001,752.000 personas (el 7,5\% de la población portuguesa) habían participado en el programa, de las cuales 354.000 (3,6\% de la población) seguían recibiendo el subsidio. Un número significativo de beneficiarios parecía haberse reinsertado con normalidad después de recibir la asistencia de la renta mínima: de las 398.000 personas que habían salido del programa, 258.000 lo habían hecho porque habían desaparecido las causas de su aguda necesidad económica. El gasto total del programa de rentas mínimas portugués alcanzó su cifra máxima de 284 millones de euros $(0,25 \%$ del PIB) en 2000 , pero descendió a 235 millones de euros $(0,19 \%$ del PIB) en 2001 . 
R I S

REVISTA INTERNACIONAL DE SOCIOLOCIA

№ 36, Septiembre-Diciembre, 2003

LUIS MORENO, MANOS MATSAGANIS, MAURIZIO FERRERA y LUIS CAPUCHA

La puesta en vigor del programa del RMG ha permitido identificar dos dificultades principales: la primera atañe a la activación laboral (incluida la calidad de los planes de inserción propiciados por las instituciones). La evidencia muestra que el fraude es insignificante, a pesar del alarmismo generado por unos pocos casos detectados. Los desincentivos laborales asociados a la percepción del subsidio son también limitados, ya que la cuantía de las prestaciones es baja y las medidas de activación cumplen como instrumentos eficaces de control. De otro lado, el programa confronta una escasez de recursos humanos dado que el enfoque personalizado, y la necesidad de supervisar el proceso complejo de inserción, constituyen una pesada carga para un número limitado de trabajadores sociales.

El RMG se convirtió en tema de debate político durante la campaña de las elecciones generales de 2002. A pesar de que la abolición del programa de rentas mínimas no figuraba en ningún programa electoral, se utilizaron argumentos tales como la necesidad de reducir el gasto público o el riesgo de la "trampa de la pobreza" para proponer cambios. La coalición de centro-derecha que había criticado duramente el programa RMG resultó la ganadora de las elecciones. Pero el nuevo gobierno aceptó el principio del derecho universal a la renta mínima, aunque subsiguientemente se han propuesto algunos cambios menores. En primer lugar, el programa se rebautizó como Rendimento Social da Inserção (RSI), según ley promulgada en mayo de 2003, a fin de enfatizar la dimensión de inserción. Se esperaban, igualmente, que los criterios de acceso al programa se hiciesen más restrictivos y se propuso la introducción de nuevos mecanismos de control del fraude. Sin embargo, el Tribunal Constitucional ha juzgado como inconstitucionales algunas de dichas propuestas, por lo que el programa mantendrá sus características esenciales al menos en el próximo futuro. Para más adelante nuevos retos podrían derivarse de las constricciones presupuestarias, las cuales podrían privar al programa de importantes recursos humanos.

\section{CONCLUSIONES}

Como nuestro precedente análisis constata, los países meridionales de la UE muestran diferencias tanto en lo referente al diseño de las políticas contra la pobreza, como a las configuraciones institucionales dentro de las cuales aquellas políticas operan. Pero continúan formando un grupo diferenciado que confronta un conjunto de retos similares y una agenda común de políticas sociales.

En contraste con el letargo característico de decenios anteriores, los años 90 han sido testigos de la puesta en marcha de significativas y prometedores políticas innovadoras en el campo de la pobreza y la exclusión social en la Europa del Sur. Nuevos subsidios y servicios selectivos se han introducido en los cuatro países en la dirección justa, es decir, han colmado algunas lagunas tradicionales 
en la cobertura social, generalmente las más macroscópicas, y han favorecido una mayor reorientación del gasto social hacia los más necesitados.

Indudablemente, las iniciativas de la UE han sido cruciales en el impulso dado a tal recalibración de políticas. La influencia de la Recomendación del Consejo 92/441 ya ha sido destacada. Los Planes de Acción Nacional para al Inclusión Social de 2001 han supuesto la primera tentativa seria de formular un diagnóstico comprensivo de los actuales retos y políticas existentes. La creciente atención hacia los "mínimos sociales" y las "mallas de seguridad" ha sido propiciada por el discurso de la UE sobre la cohesión, la inclusión y la garantía de recursos suficientes (Ferrera et al., 2002).

El proceso para establecer programas eficaces de rentas mínimas ha procedido a diferentes velocidades a través de rutas diversas en los cuatro países. Portugal ha implementado con éxito un programa nacional basado en el derecho. En España ha predominado la opción descentralizadora: en algunas Comunidades Autónomas la renta mínima es un derecho con base legal, pero, en otras, se ha adoptado un enfoque más cauto y discrecional. El programa piloto italiano se puso en marcha con la perspectiva de su posterior extensión nacional, pero su destino aparece muy incierto al no contar con recursos presupuestarios. Grecia, por su parte, no parece superar el ámbito de los debates programáticos: la opción de poner en vigor algún tipo de programa de rentas mínimas se considera todavía demasiado controvertida (tabla 3 ).

El refuerzo de la malla de seguridad de protección social suscita la cuestión de cuál es el nivel de actuación más apropiado. El asunto es particularmente relevante en el caso de España e Italia al afectar al debate de mayor calado relativo a la subsidiariedad y la descentralización. Mientras que estos últimos procesos posibilitan indudablemente nuevas oportunidades para la innovación programática y la experimentación virtuosa en el ámbito regional, pueden también perpetuar, e incluso aumentar, los tradicionales diferenciales y disparidades entre territorios. En términos generales, se puede afirmar que España parece caminar en la dirección indicada en primer lugar, mientras que el escenario en Italia apunta hacia la segunda.

Respecto al conjunto de los países meridionales de la UE, el veredicto se mantiene abierto: a pesar de los desarrollos positivos acaecidos en los años 90 , las mallas de seguridad en el sur de la UE mantienen su fragilidad, tanto en su diseño institucional, como en su apoyo político y su legitimad. Esta debilidad debería ser causa de preocupación ya que, el declinar de la tradicional disponibilidad de las familias para responder a las necesidades sociales, se corresponde con una creciente demanda por una protección formal de servicios y prestaciones sociales. Hacer frente a este desafio requerirá ciertamente un esfuerzo considerable, al menos para promover la capacidad administrativa, dado que el coste fiscal para fortalecer las mallas de seguridad continuará siendo modesto.

El futuro de las políticas contra la pobreza y, en particular, los programas de rentas mínimas dependerán, en última instancia, de consideraciones políticas. Estos 
RIS

REVISTA INTERNACIONAL DE SOCIOLOCIA

$\underline{N^{\circ} 36, \text { Septiembre-Diciembre, } 2003}$

LUIS MORENO, MANOS MATSAGANIS, MAURIZIO FERRERA Y LUIS CAPUCHA

Tabla 3.

Prestaciones de mínimos para individuos y familias (2002).

\begin{tabular}{lcccc}
\hline & España & Grecia & Italia & Portugal \\
\hline Seguro de paro (nivel mínimo) & $442^{\mathrm{a}}$ & $265^{\mathrm{a}}$ & $\mathrm{g}$ & 390 \\
Subsidio de desempleo (asistencial) & $332^{\mathrm{a}}$ & $150^{\mathrm{b}}$ & n.d. & $312^{\mathrm{d}}$ \\
Pensión mínima contributiva (70 años) & $386^{\text {ad }}$ & $474^{\mathrm{ac}}$ & $516^{\mathrm{h}}$ & 190 \\
Pensión mínima no contributiva (70 años) & $259^{\mathrm{d}}$ & $156^{\mathrm{ad}}$ & $516^{\mathrm{h}}$ & 138 \\
Subsidio familiar (1 hijo de 7 años) & 24 & n.d. $^{\mathrm{e}}$ & n.d. $^{\mathrm{e}}$ & 26 \\
Subsidio familiar (3 hijos de 10,7 y 4 años) & 73 & $141^{\mathrm{r}}$ & $110^{\mathrm{i}}$ & $* 92$ \\
\hline
\end{tabular}

Las cantidades son mensuales (en $€$ ). Las prestaciones se pagan 12 veces al año a no ser que se indique de otra manera. En su caso, los niveles pueden variar para el trabajador individual o la familia de acuerdo a los niveles de renta o de salario.

${ }^{a}$ Pagado 14 veces al año.

b Sólo disponible a trabajadores de más de 45 años.

c Incluido un suplemento de solidaridad para los pensionistas.

'Nivel para los beneficiarios sin familiares a cargo.

e No existe un programa general. Hay subsidios contributivos para las familias con trabajadores dependientes.

' Sólo disponible para familias con un tercer hijo menor de 6 años.

${ }^{g}$ El seguro ordinario de paro corresponde al $40 \%$ del salario de referencia (sin nivel mínimo).

h Pagado 13 veces al año.

i Se incluye sólo la prestación por familia numerosa. Excluidos los subsidios contributivos.

Fuente: Elaboración de los datos del informe FIPOSC y MISSOC (2002).

programas son vulnerables porque sus beneficiarios también lo son. Mantener el impulso de la reforma estriba en buena medida en la habilidad y la fuerza de las coaliciones transversales que las favorecen. Ahora sólo puede esperarse que los esfuerzos de los últimos años por coser y remendar las mallas de seguridad en la Europa meridional no sean abandonados. Los marginados de la protección social son todavía demasiados y demasiado pobres.

\section{REFERENCIAS BIBLIOGRÁFICAS}

ADDIS, E. (1999), "Gender in the reform of the Italian welfare state", South European Society \& Politics, $\mathrm{n}^{\circ}$ 4, (2), pp. 122-150.

AGUILAR, M., M. LAPARRA y M. GAVIRIA (1995), La caña y el pez: el salario social en las comunidades autónomas 1989-1994, Madrid, Foessa. 
ALMEIDA J.F., L. CAPUCHA L, A. F. COSTA, F. L. MACHADO, I. NICOLAU y E. REIS (1994), Exclusão social, factores e tipos de pobreza em Portugal, Oeiras, Celta Editora.

ARRIBA, A. (2002), "Procesos de implantación de políticas de rentas mínimas de inserción en España", en Moreno, L. (ed.), Pobreza y exclusión: la "malla de seguridad" en España, Madrid, CSIC.

ARRIBA, A. y L. MORENO (2002), Poverty, social exclusion and "safety nets", UPC Working, Paper, 02-10, Madrid (www.iesam.csic.es/doctrab).

ATKINSON, A. (1998), Poverty in Europe, Oxford, Blackwell.

BALDINI, M., P. BOSI y S. TOSO (2002), "Targeting welfare in Italy: old problems and perspectives on reform", Fiscal Studies, $\mathrm{n}^{\circ} 23$ (1), $\mathrm{n}^{\circ}$ 51-75.

BALDWIN-EDWARDS, M. y J. ARANGO (ed.) (1998), "Immigrants and the informal economy in southern Europe", South European Society \& Politics, $n^{\circ} 3$ (3), (número monográfico).

BENASSI, D. (2001), Le politiche nazionali contro la povertà in Italia. Rapporto per la Commissione di indagine sull'esclusione sociale, Roma, Presidenza del Consiglio dei Ministri.

CAPUCHA, L. (1998), Rendimento Minimo Garantido: avaliação da fase experimental, Lisboa, CIES/MTS.

CEC (2001), Draft joint report on social inclusion. Communication from the Commission, COM (2001) 565 final.

(2002), The social situation in the European Union, Luxemburgo, Oficina de publicación de las comunidades Europeas.

ESPING-ANDERSEN G., D. CON GALLIE, A. HEMERIJCK y J. MYLES (2002), Why we need a new welfare state, Oxford, Oxford University Press.

FERNÁNDEZ CORDÓN, J. A. (1997), "Youth residential independence and autonomy: a comparative study", Journal of Family Issues, n 6, pp. 576-607.

FERRERA, M. (1995), "Los Estados del Bienestar del Sur en la Europa social”, en Sarasa, S. y Moreno, L. (eds.), El Estado del Bienestar en la Europa del Sur, pp. 85-111, Madrid, CSIC.

FERRERA, M., A. HEMERIJCK y M. RHODES (2000), The future of social Europe: recasting work and welfare in the new economy, Oeiras, Celta Editores.

FERRERA, M., M. MATSAGANIS y S. SACCHI (2002), “Open co-ordination against poverty: the new EU 'social inclusion process"', Journal of European Social Policy, $\mathrm{n}^{\circ} 12$ (3), pp. 227 239.

GOUGH, I. (1996), "Social assistance in Southern Europe", South European Society \& Politics, $\mathrm{n}^{\mathrm{o}} 1$ (1), pp. 1-23. 
R I S

REVISTA INTERNACIONAL DE SOCIOLOCIA

№ 36, Septiembre-Diciembre, 2003

LUIS MORENO, MANOS MATSAGANIS, MAURIZIO FERRERA Y LUIS CAPUCHA

GUILLÉN A. y M. MATSAGANIS (2000), "Testing the 'social dumping hypothesis in southern Europe: welfare policies in Greece and Spain during the last 20 years", Journal of European Social Policy, 10 (2): 120-145.

IRS, FONDAZIONE ZANCAN y CLES (2001), Valutazione della sperimentazione del Reddito Minimo di Inserimento, Roma, Presidenza del Consiglio dei Ministri.

KAUFMANN, D., A. KRAAY y P. ZOIDO-LOBATÓN (2002), Government Matters II: Updated Indicators for 2000/2001, The World Bank Institute (www.worldbank.org/wbi/governance/pdf/ govmatters2.pdf).

LEIBFRIED, S. (1993), "Towards a European welfare state?", en C. Jones (ed.), New perspectives on the welfare state in Europe, Londres, Routledge.

LEWIS, J. (2001), "The decline of the male breadwinner model: the implications for work and care", Social Politics, $\mathrm{n}^{\circ} 8$ (2), pp. 152-70.

MARLIER, E. y M. COHEN-SOLAL (2000), "Social benefits and their redistributive effect in the EU", Statistics in Focus (Theme 3 9/2000), Luxemburgo, Eurostat.

MATSAGANIS, M. (2002), 'Yet another piece of pension reform in Greece', South European Society \& Politics 7 (3): 109-122.

MATSAGANIS, M., F. PAPADOPOULOS y P. TSAKLOGLOU (2001), "Eliminating extreme poverty in Greece", Journal of Income Distribution, $n^{o} 10$ (1-2), pp. 40-57.

MISSOC (2002), Social protection in the EU member states and the European Economic Area (www.europa.eu.int/comm/employment_social/missoc).

MORENO, L. (1997), La federalización de España. Poder politico y territorio, Madrid, Siglo XXI.

(2002), "Bienestar mediterráneo y supermujeres”, Revista Española de Sociología, nº 2, pp. 41-57.

(2003), "Europeanisation, mesogovernments and safety nets", European Journal of Political Research, $\mathrm{n}^{\circ} 42$, (2), pp. 185-199.

ONOFRI (INFORME) (1997), Commissione per l'analisi delle compatibilità macroeconomiche della spesa sociale (Relazione Finale), Roma, Presidenza del Consiglio dei Ministri.

RHODES, M. (1996) "Southern European welfare states: identity, problems and prospects for reform", South European Society \& Politics, n 1, (3), pp. 1-22.

RODRÍGUEZ-CABRERO, G., A. ARRIBA y V. MARBÁN (2003), Reformas del bienestar en España: Informe basado en mapas de politicas públicas (Spain's National Report on Policy Maps of Welfare Reform), Madrid: Working Paper UPC 03-11 (www.iesam.csic.es/doctrab.htm).

SARACENO, C. (2000), "Italian families under economic stress: the impact of social policies", Labour, $\mathrm{n}^{\circ} 14$ (1), pp. 161-183.

(2002), Social assistance dynamics: national and local poverty regimes. Bristol, Policy Press.

30 
SARASA, S. y L. MORENO (EDS.) (1995), El Estado del Bienestar en la Europa del Sur, Madrid, CSIC.

TAYLOR-GOOBY, P. (ED.) (2001), Welfare states under pressure, Londres, Sage.

VIEGAS, J. y A. F. COSTA (2000), Crossroads to modernity: contemporary Portuguese society, Oeiras, Celta Editora. 